\title{
Aiding reduction of smoking with nicotine replacement medications: hope for the recalcitrant smoker?
}

Karl Olov Fagerström, Ronny Tejding, Åke Westin, Erik Lunell

\begin{abstract}
Objective-To assess the effect of the various nicotine replacement therapies (NRT) on smoking reduction.

Design-During an initial sampling week, the subjects familiarised themselves with nicotine gum, patch, nasal spray, vaporiser (vapour inhaler) and sublingual tablet. A crossover design was used during the next four study weeks; during two of these weeks the subjects could select one nicotine replacement product of their choice to use, whereas during the other two they were randomly assigned a product to use.
\end{abstract}

Subjects-143 men and women smoking an average of 22.6 (SD 7.0) cigarettes per day and exhibiting a Fagerström Tolerance Questionnaire (FTQ) score of 7.0 (SD 1.9).

Interventions-Subjects were asked to use as much NRT as they wished, yet to smoke enough to feel comfortable.

Main outcome measures-Self-reported cigarette consumption, exhaled carbon monoxide (CO), withdrawal symptom score, cotinine plasma levels and motivation to quit were monitored over a period of five weeks.

Results-Self-reported smoking declined steadily over the five weeks, from 22.6 (SD 7.0) to $10.4(S D 1.0)(P<0.001)$ cigarettes daily $(54 \%$ decrease), with the biggest drop $(37 \%)$ during the first productsampling week. Smoking reduction was greater on average during the weeks when the subjects could choose their nicotine product than when products were assigned. CO readings decreased from 22.7 (SD 8.5) to 14.8 (SD 8.4) $\mathrm{ppm}$ $(\mathbf{P}<0.001)$ confirming a reduction in smoking (35\% decrease), although cotinine levels remained steady, suggesting that subjects were titrating nicotine to their original levels. Withdrawal scores decreased over time $\mathbf{( 3 2 \%}$ decrease, $\mathbf{P}<0.001$ ), showing that there was no discomfort associated with the smoking reduction, and motivation to quit was enhanced by the treatment in most subjects $(93 \%)$.

Conclusions-NRT for aiding smoking reduction appeared to be safe, was associated with a clinically significant reduction in smoke exposure over a five-week follow up, and increased motivation to stop smoking. A smoking reduction procedure may help the very recalcitrant smoker gain confidence and increase the control over his/her smoking behaviour. More controlled research is needed to follow up these promising results.

(Tobacco Control 1997;6:311-316)

Keywords: nicotine, nicotine replacement therapy, smoking reduction

\section{Introduction}

Smoking tobacco is a cause of several types of cancer as well as cardiovascular and respiratory disease. As many as half of long-term smokers are prematurely killed by their smoking addiction. ${ }^{1}$ For most disorders there is a doseresponse relationship - that is, the heavier the cigarette consumption, the larger the risk. ${ }^{1}$ The relative risk of death from chronic obstructive pulmonary disease (COPD) rises to 4.2 for smokers smoking fewer than 10 cigarettes per day and to 18.3 for those smoking more than 40 cigarettes per day, compared with non-smokers. ${ }^{2}$ Fortunately, giving up smoking produces a substantial risk reduction. ${ }^{34}$

Many smokers would like to give up smoking, but for most of them it is difficult to quit, and therefore only about half of smokers succeed in quitting during their lifetime. In the management of patients with smoking-related disorders such as COPD, concern has been expressed over the large majority of smokers who cannot give up this disease-producing behaviour. Because higher cigarette consumption levels are associated with a higher risk of respiratory impairment (such as loss of $\mathrm{FEV}_{1}$ ) and the development of COPD, ${ }^{56}$ physicians have often advised such patients to reduce their cigarette consumption. Unfortunately, reduced smoking has seldomly lasted. One exception was a group of very heavy smokers treated with behavioural methods for smoking reduction that showed significant reduction after 2.5 years of follow up. ${ }^{7}$ In a more recent population-based study, it was found that $80 \%$ of those trying to reduce their smoking were unable to do so. ${ }^{8}$

It has now been established that tobacco smoking often involves a dependence to nicotine. ${ }^{9}$ This is usually seen as a problem, but it also offers an opportunity. The cigarette is the most contaminated drug-delivery device. ${ }^{10}$ If nicotine is largely what the smoker needs, the use of nicotine from alternative sources to sustain smoking reduction could be exploited for long-term harm reduction to the smoker \\ Consumer Healthcare,
Helsingborg, Sweden \\ am.pnu.com
}


unable to quit. The first study to test this idea was in heavy smokers with COPD, where a substantial reduction in smoking was found, resulting in reduced bronchial inflammation when nicotine gum was substituted for cigarettes. ${ }^{11}$ In a second study, the same group of investigators found that the amount of reduced smoking correlated with the number of pieces of gum used, but that gum overall produced a non-significant trend in reduced smoking. ${ }^{12}$ Smoking reduction could possibly also work as an intervention before smoking cessation attempts, by improving success rates in recalcitrant smokers.

Today several different nicotine replacement therapies (NRT) are available, and more are under development. ${ }^{13}$ From the standpoint that there is a medical need for reduced smoking among patients with smoking-related disorders who cannot or do not want to give up smoking, the issue of whether NRT can reduce cigarette consumption among continuing smokers is clinically important. The purpose of this study was to determine whether smokers would reduce their cigarette use during a relatively extended period of time (five weeks) if they were allowed to use one or more nicotine replacement products simultaneously with smoking. The following main issues were addressed.

- Is stable smoking reduction over five weeks possible if nicotine intake is supplemented from NRT?

- Is a personal choice of medication important for achieving a better effect?

- Is motivation for complete cessation influenced by the opportunity to reduce smoking?

\section{Methods}

DESIGN

The study had an open crossover design, where one condition was randomisation to a specific nicotine replacement (NR) medication (gum, patch, nasal spray, vaporiser, or tablet) and the other condition was a free choice of the preferred medication. The order between the conditions was balanced. Each of the two conditions lasted two weeks. Before these two conditions there was a one-week period when the subjects could familiarise themselves with the different NRT medications - that is, use each of them for a whole day (figure 1).

\section{SUBJECTS}

Subjects were contacted through a local newspaper advertisement. The message was that if they did not want to or could not give up

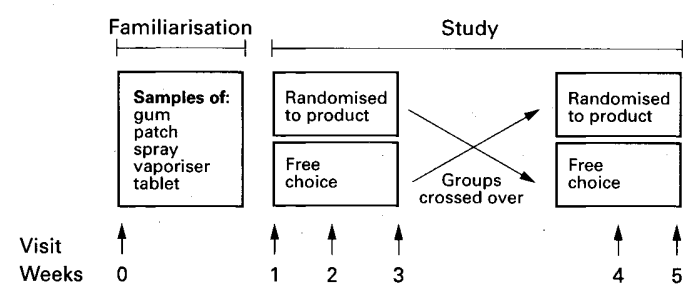

Figure 1 Design of the smoking reduction study. Weeks 2-3 comprise phase 1 of the study and weeks 4-5 comprise phase 2. Numbers indicate completion of each week. smoking, they were welcome to participate in a study where they were to reduce their smoking with the help of NR medications. All subjects signed an informed consent. The study was approved by the ethical committee of the University of Lund and was conducted at the Pharmacia \& Upjohn Clinical Pharmacology Unit in Lund, Sweden.

Subjects had to meet the following criteria: smoking a minimum of 15 cigarettes per day (average daily consumption in Sweden is 13 cigarettes per day), at least 20 years old, healthy and not on any medication, not on any NRT, not smoking cigars or pipes, and not using smokeless tobacco. A total of 170 subjects were enrolled, but data are reported from $143(84 \%)$ who provided complete information.

\section{INTERVENTIONS}

After answering the advertisement, the subjects were invited to the clinic for a preliminary screening. At the first visit, inclusion and exclusion criteria were checked. Information, verbal and written, was given about the study procedures. Assessments were made and instructions on how to use the different NR medications were given verbally and in writing. Subjects were given samples of each of the different NR medications to try during the first week along with daily recording of cigarettes smoked (which continued for the whole study). All visits were separated by one week.

All NR medications except the tablet are available on the market. The tablet contains $2 \mathrm{mg}$ nicotine and has similar pharmacokinetics as the $2 \mathrm{mg}$ Nicorette gum. Subjects were instructed to keep it still under the tongue or at any other place between the gum and cheek. It dissolves in approximately 20 minutes.

At visit 2, the first phase of the study started. Subjects were now randomly allocated to either receive their medication of choice or their randomly assigned medication. They were now asked explicitly to try to smoke less, to use as much NR medication as they wished (within a certain upper limit), but at all occasions to smoke enough to feel comfortable, thus not straining themselves.

At visit 3 (in the middle of phase 1), the subjects visited the clinic for a checkup of any problems associated with the assessments (see "Main outcome measures" below).

At visit 4, subjects switched to the second phase. At this visit they evaluated the NR medication used during their first phase. Visits 5 and 6 were for assessment of the converse condition (figure 1). At visit 6 (study termination), subjects also evaluated the reduced smoking procedure for its potential as a smoking cessation method and reported whether their motivation to stop smoking had changed as a result of their study participation.

MAIN OUTCOME MEASURES

Assessments made were number of cigarettes smoked per day, carbon monoxide in exhaled air (CO) analysed by a smokelyser (Bedfont), saliva cotinine concentration, nicotine dependence by the Fagerström Tolerance Questionnaire 
Table 1 Demographic characteristics of participating subjects in the free-choice $(n=74)$ and randomised $(n=69)$ conditions

\begin{tabular}{lllllr}
\hline & \multicolumn{2}{c}{ Mean (SD) } & & \multicolumn{2}{c}{ Range } \\
\cline { 2 - 3 } & Free choice & Randomised & & Free choice & Randomised \\
\hline Age (years) & $46.7(11.3)$ & $44.7(12.6)$ & & $26-77$ & $24-73$ \\
FTQ & $6.9(2.1)$ & $7.1(1.7)$ & & $2-11$ & $3-11$ \\
Cigarettes/day & $22.6(7.0)$ & $22.7(7.0)$ & & $15-50$ & $15-60$ \\
Years smoked & $28.0(11.2)$ & $27.4(11.6)$ & & $3-53$ & $8-58$ \\
Men (\%) & 35 & & & & \\
Women (\%) & 65 & 60 & & \\
Tried to quit (\%) & 97 & 86 & & & \\
Tried to smoke less (\%) & 81 & 77 & & & \\
\hline
\end{tabular}

$\mathrm{SD}=$ standard deviation.

FTQ = Fagerström Tolerance Questionnaire.

Table 2 Cigarettes per day, exhaled carbon monoxide (CO), total withdrawal score, and saliva cotinine concentrations during the study $(n=143)$

\begin{tabular}{lllll}
\hline & \multicolumn{4}{c}{ Mean (SD) } \\
\cline { 2 - 5 } Visit & Cigarettes/day & $\begin{array}{l}\text { Exhaled CO } \\
(p p m)\end{array}$ & $\begin{array}{l}\text { Cotinine } \\
(\mathrm{ng} / \mathrm{ml})\end{array}$ & $\begin{array}{l}\text { Total withdrawal } \\
\text { score }\end{array}$ \\
\hline Screening & $22.6(7.0)$ & $22.7(8.5)$ & $360(134)$ & $13.5(9.3)$ \\
Week 1 & $14.3(7.5)$ & $18.4(8.7)$ & $347(136)$ & $12.5(8.1)$ \\
Week 2 & $12.3(7.0)$ & $16.4(7.9)$ & $364(168)$ & $11.4(7.9)$ \\
Week 3 & $11.5(7.1)$ & $16.1(8.1)$ & $350(158)$ & $10.3(8.3)$ \\
Week 4 & $10.6(5.9)$ & $14.8(7.5)$ & $373(174)$ & $9.7(7.6)$ \\
Week 5 & $10.4(5.6)$ & $14.8(8.3)$ & $349(160)$ & $9.0(7.7)$ \\
\hline
\end{tabular}

*Based on the sum of 17 withdrawal symptoms assessed (craving, irritation, restlessness, concentration difficulties, impatience, sweating, anger, depression, drowsiness, anxiety, headache, insomnia, tremor, sleepiness, hunger, increased food intake, and dizziness). See text for details.

FTQ, ${ }^{14}$ and withdrawal symptoms (craving, irritation, restlessness, concentration difficulties, impatience, sweating, anger, depression, drowsiness, anxiety, headache, insomnia, tremor, sleepiness, hunger, increased food intake, and dizziness). The subjects rated each symptom on a five-point scale at all visits. The absence of symptoms was scored 0 , and the highest magnitude was scored 4 . The total withdrawal score was arrived at by adding the scores for each individual symptom, which gives a minimum score of 0 , and a maximum score of $68(4 \times 17)$.

At all visits, daily NR medication use, number of cigarettes smoked, saliva cotinine, and exhaled $\mathrm{CO}$ were recorded and an evaluation of the withdrawal symptoms and NR medication liking were made. Subjects were asked about adverse effects at each visit, and onset, duration, and severity (graded mild, moderate, or severe) were recorded.

At the last visit the subjects also rated the influence that participation in the smoking reduction study had exerted on their motivation to give up smoking, and whether reduced smoking is a good method to give up smoking.

DATA ANALYSIS PROCEDURES

Due to the explorative character of the study, no formal sample size calculations were done. For outcome variables, differences between weeks 4 and 2 and between week 2 and baseline were computed for each subject. These were analysed using analyses of variance, with treatment and order (randomised and free choice) as independent variables. Trends were analysed using linear regression. Descriptive statistics and tabula- tions were used for baseline variables. All significance tests are two sided and at a 5\% level.

\section{Results}

DEMOGRAPHICS

Demographic characteristics of the 143 smokers are shown in table 1 . The subjects included were rather nicotine dependent. Their mean cigarette consumption of 22.6 (SD 7.0) per day and their mean FTQ of 7.0 (SD 1.9) should be compared with the Swedish smoking population's average of 14 cigarettes per day and an FTQ of $4.2 .{ }^{15}$ No differences in baseline data were found between those starting with free choice $v s$ randomised to medication.

\section{NUMBER OF CIGARETTES}

Number of cigarettes smoked per day is shown in table 2 . In the run-in week, when the five different medications were tried, a 37\% reduction in smoking was occurring, from 22.6 (SD 7.0) cigarettes per day at inclusion to 14.3 per day when the experiment started $(P<0.001)$. During the first two weeks (phase 1), smoking further declined to 11.5 cigarettes per day $(\mathrm{P}<0.001)$. At four weeks (at the end of the second phase), subjects were smoking 10.4 (SD 1.0) cigarettes per day, which is $46 \%$ of baseline $(\mathrm{P}<0.001)$. Fifty-nine per cent reduced their smoking by more than $50 \%$ and, among these, 5\% smoked no cigarettes and had no excess $\mathrm{CO}$ at the end of four weeks.

\section{CARBON MONOXIDE IN EXHALED AIR}

The data for exhaled carbon monoxide measurements are shown in table 2. At baseline, an average CO reading of 22.7 (SD 8.5) ppm was obtained. This was reduced by $19 \%$ to $18.4 \mathrm{ppm}$ at the end of the run-in period $(P<0.001)$. At the end of the first two weeks of controlled smoking, a further reduction to $16.1 \mathrm{ppm}$ was seen $(P<0.001)$, and at the end of the second phase $\mathrm{CO}$ further declined to 14.8 (SD 8.3 ) ppm (65\% of baseline). There were no differences between the five medications in their effect in decreasing exhaled $\mathrm{CO}$ and the number of cigarettes smoked per day (table 3 ).

\section{SALIVA COTININE CONCENTRATION}

The values at the initial screening visit, at the start of reduced smoking, and at three and five weeks, were $360,347,350$, and $349 \mathrm{ng} / \mathrm{ml}$, respectively (table 2 ). There was no significant difference between any time points.

\section{WITHDRAWAL SYMPTOMS}

In table 2 the 17 symptoms are summarised into a total withdrawal score (TW). From the screening visit and onwards there is a steady decline in total withdrawal score (linear regression, $F=16.7, \mathrm{P}<0.001$ ). Of the 17 symptoms, a significant decline in occurrence from the start to the end of the experiment was seen for restlessness, difficulties concentrating, impatience, irritation, and sweating $(\mathrm{P}<0.001)$; and for anger and depression $(P<0.05)$. No change was seen for tremor, sleep, hunger, food intake, 


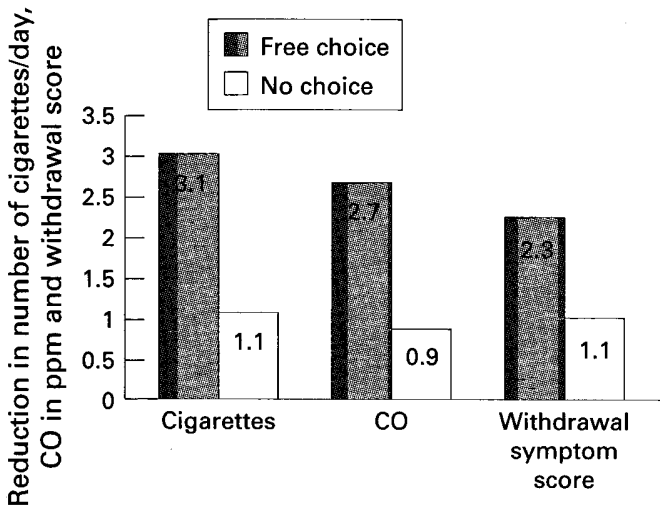

Figure 2 Effect of choice of medication compared with random allocation to medication on reduction of cigarettes per day, exhaled carbon monoxide (CO), and total withdrawal symptom score (see text for definition).

and dizziness. TW at the screening visit, and at weeks 1,3 , and 5 , was $13.5,12.5,10.3$, and 9.0 , respectively.

\section{EFFECT OF FREE CHOICE OF NICOTINE}

MEDICATION

Daily use of medication was reported by $93 \%$ of the subjects when in the free choice condition vs $88 \%$ when randomised to medication (not significant). When starting the formal experiment the $\mathrm{CO}$ levels of those given free choice $v s$ random assignment were 18.7 and $18.1 \mathrm{ppm}$, respectively (not significant). During the first phase the free-choice subjects showed a drop in CO level of $2.7 \mathrm{ppm} v s$ $1.8 \mathrm{ppm}$ for the no-choice group (not significant). At the end of the second phase the corresponding figures were 2.6 vs $0.1 \mathrm{ppm}$ (not significant). The overall effect of choice on $\mathrm{CO}$ reduction (combining the data from both phases) was 2.7 vs $0.9 \mathrm{ppm}(\mathrm{P}<0.05$, figure 2$)$.

When starting the formal experiment the daily cigarette consumption of those given free choice was 14.3 vs 14.4 for those with no choice. During the first phase, free-choice subjects had a drop in cigarette consumption of 3.8 cigarettes vs 2.2 for the no-choice group $(P<0.05)$. In the end of the second phase, the corresponding figures were 2.5 vs 0.02 cigarettes per day $(P<0.01)$. The overall effect of free choice on reduction of daily cigarette consumption was 3.1 cigarettes vs 1.1 ( $P<0.001$, figure 2).

At the start of the experiment, the cotinine concentrations of free choice vs no choice were 348 vs $346 \mathrm{ng} / \mathrm{ml}$ (not significant). At the end of the first phase of the study, the corresponding values were 342 vs $360 \mathrm{ng} / \mathrm{ml}$ (not significant). At the end of the second phase, the free-choice subjects had cotinine concentrations of $365 \mathrm{ng} / \mathrm{ml}$ and the no-choice subjects $336 \mathrm{ng} / \mathrm{ml}$ (not significant). The overall effect of free choice $v s$ no choice regarding change in cotinine concentrations was +1.6 vs $-1.7 \mathrm{ng}$ / $\mathrm{ml}$ (not significant).

At the start of controlled smoking, the total withdrawal score for free choice $v s$ no choice was 13.4 and 11.5 points, respectively (not significant). During the first phase, the free-choice group had a drop in total withdrawal of 3.2 points $v s 1.0$ for no-choice group (not significant). In the second phase, the corresponding figures were 1.4 vs 1.2 points (not significant). The overall effect of free choice and no choice on total withdrawal reduction was 2.3 vs 1.1 points (not significant, figure 2).

PREFERENCE FOR THE VARIOUS NR MEDICATIONS If all medications had been equally preferred, they should each have had a $20 \%$ selection. Preference actually centred around $20 \%$, with the most preferred product chosen by $24 \%$ of participants, and the least preferred chosen by $15 \%$. Thus medication preference was fairly evenly distributed among the five products (table 3). Table 3 also shows how each medication was judged with respect to staying off cigarettes, reduction of craving, value for cessation, and similarity to cigarettes. Patch and vaporiser seemed not to be as good in reducing craving as gum and spray, and spray was rated most similar to cigarettes.

MOTIVATION FOR CONTROLLED SMOKING AND COMPLETE CESSATION

Motivation for reducing smoking was at inclusion very strong, averaging 8.7 (SD 1.6) points on a 10-point scale. At the last visit subjects were asked whether their experience from the present study had changed their attitude towards the possibility of giving up smoking altogether. One per cent said that their possibility to give up had diminished, $6 \%$ reported no change, and $93 \%$ stated that they were more likely to give up smoking as a consequence of this study. At the same visit, they were asked to rate whether reduction is a good method for them to quit smoking. Ninety-two per cent said "yes" and 8\% "no". It is also noteworthy that seven subjects actually gave up smoking during the course of the study, although they were not expected to do so. A common explanation was that it was easier than they had anticipated. Six of the

Table 3 Efficacy and evaluation of the nicotine medications

\begin{tabular}{lccccc}
\hline & Patch & Gum & Tablet & Vaporiser & Nasal spray \\
\hline Decrease from start of phase I to end of phase II (mean (SD)) & & & & \\
$\quad$ Exhaled CO (ppm) & $3.9(7.4)$ & $2.9(7.0)$ & $3.1(5.9)$ & $3.9(8.1)$ & $4.4(7.3)$ \\
Cigarettes/day & $4.0(5.2)$ & $4.0(7.6)$ & $4.9(6.7)$ & $2.1(3.4)$ & $4.5(4.5)$ \\
& & & & \\
Preference in free-choice condition (\%) & 20.6 & 16.3 & 24.1 & 14.9 & 24.1 \\
& & & & & \\
Rated medications as very good or extremely good (\%) in & 31 & 40 & 41 & 37 & 36 \\
Staying off cigarettes & 15 & 48 & 41 & 23 & 45 \\
Reducing craving & 58 & 56 & 64 & 50 & 58 \\
Smoking cessation & 0 & 12 & 16 & 14 & 32 \\
Similarity to cigarettes & & & & \\
\hline
\end{tabular}


seven subjects were able to be contacted for a six-month follow up, and three stated that they were still non-smokers (not CO verified).

AMOUNT OF MEDICATION USED

Across time and conditions, the daily number of doses used for patch, gum, tablet, vaporiser, and spray were $1.1,8.5,12.0,5.8$, and 9.9. With respect to condition, there was no difference in the number of doses used for each individual medication; but when totalling all medications except patch, more was used in the free-choice condition, 37.4 vs 32.7 doses per week $(P<0.01)$. One vaporiser cartridge was counted as one dose.

\section{ADVERSE EFFECTS}

The most common adverse effect was a mild tingling effect (paraesthesia) in various parts of the body, with 20 reports in the free choice vs 24 in the randomised treatment period. Other, less frequent adverse effects reported were throat irritation and gastritis, particularly for the chewing gum and the tablet; throat irritation and coughing for the vaporiser; and headache and sore nose for the nasal spray. All adverse effects were mild to moderate; there were no reports of severe intensity. There were no serious adverse effects reported.

\section{Discussion}

We found that the number of cigarettes smoked was steadily reduced over five weeks from screening to the end of the experiment. The steady decline was also seen for exhaled $\mathrm{CO}$ and withdrawal symptoms. The lowest values were observed at study termination. Compared with baseline, the reductions were 54\% for smoking, $35 \%$ for $\mathrm{CO}$, and $32 \%$ for withdrawal symptoms. This is very encouraging because reduction in smoking without NR medication support has been difficult to maintain over time ${ }^{16}$ with one notable exception. ${ }^{7}$ The reduction in most withdrawal symptoms suggests that the subjects complied well with the instruction to smoke enough to feel all right, but they also used enough NR medication to be able to halve their cigarette consumption.

The decrease in number of cigarettes smoked was somewhat larger than the decrease in $\mathrm{CO}$, as also seen elsewhere, ${ }^{12}$ suggesting that subjects either reported fewer cigarettes than actually smoked or that they smoked the remaining ones more intensively. It would not have been surprising if a $54 \%$ reduction in cigarette smoking had caused an increase in craving and other withdrawal symptoms. In one study by Hatsukami et $a{ }^{17}{ }^{17}$ withdrawal symptoms were somewhat elevated after a $50 \%$ reduction, but not nearly as much as with abstinence. Had not the cotinine concentrations in our study been so remarkably stable, one might have speculated that a larger nicotine intake to decrease withdrawal had occurred.

A higher nicotine intake, which is sometimes seen when NR medications and smoking are combined, ${ }^{18}$ was evidently not the case in our study, even though some of the NR medications (gum, vaporiser, and tablet) produce more cotinine per nicotine unit due to swallowing and first-passage metabolism. The concomitant use of NR products and cigarette smoking could theoretically produce high plasma nicotine concentrations and consequently subjective and objective-for example, haemodynamic - effects. There are few reports on the combined effects of NRT and smoking in the literature. Foulds et al reported higher nicotine concentrations with combined use but no serious symptoms of overdose. ${ }^{18}$ In one study on the safety of transdermal nicotine in 156 patients with coronary artery disease, patients with the highest plasma nicotine levels (due in most cases to the effects of cigarette smoking concomitantly with patch use) did not appear to have an increased incidence of adverse effects. ${ }^{19}$ The safety of nicotine gum used concomitantly with smoking was assessed in the American five-year Lung Health Study, ${ }^{20}$ and the incidence of side effects among the concomitant users ( $\mathrm{n}=389$ )-mainly dizziness, headache, and throat irritation-did not differ significantly from that among 2554 nonsmoking nicotine gum users.

The clinical situation that would most closely resemble a worst-case scenario of concomitant use of NRT and smoking is the aversive procedure described as "rapid smoking" (RS). The standard RS procedure requires the subject to inhale from a cigarette once every six seconds until nauseated. ${ }^{21}$ Sachs et al found that RS produced statistically significant increases in heart and respiratory rates, systolic blood pressure, and $\mathrm{CO}$ in healthy, young, male smokers. ${ }^{22}$ They found no electrocardiographic abnormalities contrary to a earlier report. $^{23}$ In 21 patients with cardiopulmonary disease trying RS, there was no evidence of myocardial ischaemia or significant cardiac arrhythmia. ${ }^{24}$ Particularly heavy smokers regulate their plasma nicotine concentrations well and therefore substantially increased concentrations with concomitant smoking and use of NRT are unlikely to occur. ${ }^{25}$

In a recent study of cardiac patients who were given active or placebo patches and who smoked at the same time, it was found that the circulation in the myocardium was improved with active patch. This was most likely due to reduced smoking and $\mathrm{CO}$ concentrations. ${ }^{26}$

There was no placebo control for the NR medications used in this experimental design and therefore conclusions that the trend over time was not simply an instructional effect cannot be made. Smoking behaviour can be highly reactive, but a decrease in number of cigarettes smoked is usually maintained for only a short time. ${ }^{27}$ The participating subjects were markedly nicotine dependent, which should have made it more difficult to reduce smoking for a longer time period. ${ }^{25}$ The relatively long-lasting and steady decline in smoking thus suggests that the findings are not only a result of instruction. However, more controlled research is needed to follow up on these promising results. 
What can be more firmly concluded from the results of this experiment is that a larger reduction in smoking, exhaled $\mathrm{CO}$, and withdrawal was obtained when the subjects could choose the medications they preferred after having tried them. The steepest decline was actually seen during the run-in week when subjects were expected to try all of the medications. It is not known whether they used more NR medication at that time - for example, in combinations - than later when they had access to only one medication at a time.

The large reduction seen during the run-in week could also have occurred because it would be easier to reduce from the highest number of cigarettes smoked than later, after some reduction already had taken place. A larger reduction in smoking could possibly be achieved if subjects were asked to try harder and accept some discomfort. Few differences among the NR medications were noted, and the degree of preference was relatively similar for each. However, individual subjects showed strong preference for one or two medications, which makes an effective choice possible.

In conclusion, a clinically significant reduction in smoke exposure occurred and was maintained for five weeks. NRT for aiding reduction appeared to be safe, and reducing smoking did not undermine but in fact increased motivation to stop smoking. Specifically, $93 \%$ of the subjects found that the experience from the study had made them more motivated to quit smoking. Thus, a smoking reduction procedure may help the very recalcitrant smoker gain confidence and increase the control over his/her smoking behaviour. The effect of a gradual cessation procedure to achieve complete cessation for the recalcitrant smoker needs to be further investigated.

At the time when this study was carried out, all authors were employees of Pharmacia \& Upjohn, which manufactures nicotine replacement products.

1 Doll R, Peto R, Hall E, Wheatley K, Gray R. Mortality in relation to consumption of alcohol: 13 years' observations on male British doctors. BMF 1994;309:911-18.

2 Higgins MW. Risk factors associated with chronic obstructive lung disease. Ann NY Acad Sci 1991;624:7-17.

3 US Department of Health and Human Services. The health benefits of smoking cessation. A report of the Surgeon General, benefits of smoking cessation. A report of the Surgeon General, 1990. Rockville, Maryland: Public Health Service, Centers for Disease Control, Office on Smoking and

4 Anthonisen NR, Connett JE, Kiley JP, et al. Effects of smoking intervention and the use of an inhaled anticholinergic bronchodilator on the rate of decline of $\mathrm{FEV}_{1}$ - the Lung Health Study. $¥ A M A$ 1994;272:1497-505.
5 Camilli AE, Burrows B, Knudson RJ, Lyle SK, Lebowitz $\mathrm{MD}$. Longitudinal changes in forced expiratory volume in one second in adults: effects of smoking and smoking cessation. Am Rev Respir Dis 1987;135:794-9.

6 Kuller LH, Ockene JK, Townsend M, Browner W, Meilahn $\mathrm{E}$, Wentworth DN. The epidemiology of pulmonary function and COPD mortality in the Multiple Risk Factor Intervention Trial. Am Rev Respir Dis 1989;140:S78-81.

7 Glasgow RE, Klesges RC, Klesges LM, Vasey MW, Gunnarsson DF. Long term effects of a controlled smoking program: a $2 \frac{1 / 2}{2}$ year follow-up. Behav Ther 1985 16:303-7.

8 US Centers for Disease Control and Prevention. Indicators of nicotine addiction among women-United States, 1991-1992. MMWR 1995;44:102-5.

9 World Health Organisation. International classification of mental and behavioural disorders. Geneva: WHO, 1992

10 Henningfield JE, Keenan RM. The anatomy of nicotine addiction. Health Values 1993;17:12-19.

1 Rennard SI, Daughton D, Fujita J, et al. Short-term smoking reduction is associated with reduction in measures of lowe respiratory tract inflammation in heavy smokers. Eur Respir F 1990;3:752-9.

12 Rennard SI, Daughton DM, Thompson AB, Fioreani AA Romberger DJ, Millatmal $\mathrm{T}$. The effects of nicotine replacement therapy on cigarette smoking reduction (abstract). American Lung Association/American Thoracic Society international conference. Boston, Massachusetts: Society international

13 Tang JL, Law $M$, Wald N. How effective is nicotine replacement to stop smoking? BMF 1994;308:21-6.

14 Heatherton TF, Kozlowski LT, Frecker RC, Fagerström $\mathrm{K}-\mathrm{O}$. The Fagerström test for nicotine dependence: a revision of the Fagerström Tolerance Questionnaire. $B r \mathcal{F}$ Addict 1991;86:1119-27.

15 Ramström L. ITS-96. Smoking survey. Stockholm: Institute for Tobacco Studies, 1997.

16 Schmitz JM, Rhoades H, Grabowski J. Contingent reinforcement for reduced carbon monoxide levels in methadone maintenance patients. Addict Behav 1995;20:171-9.

17 Hatsukami DK, Dalgren L, Hughes JR. Symptoms of tobacco withdrawal from abrupt cigarette cessation versus partial cigarette reduction. Psychopharmacology 1988 ; 94:242-7.

18 Foulds I, Stapleton J, Feyerabend C, Vesey C, Jarvis M, Russell MA. Effect of transdermal nicotine patches on cigarette smoking: a double blind crossover study. Psychopharmacology 1992;106:421-7.

19 Working group for the study of transdermal nicotine in patients with coronary artery disease. Nicotine replacement therapy for patients with coronary artery disease. Arch Intern Med 1994;154:989-95.

20 Murray RP, Bailey WC, Daniels K, et al. Safety of nicotine polacrilex gum used by 3094 participants in the Lung polacrilex gum used by 3094 participar

21 Lichtenstein E, Glasgow RE. Rapid smoking: side effects and safeguards. $\mathcal{F}$ Consult Clin Psychol 1977;45:815-21.

22 Sachs EPL, Hall RG, Hall SM. Effects of rapid smoking: physiologic evaluation of a smoking cessation therapy. $A n n$ Int Med 1978;88:639-41.

23 Horan JJ, Hacket G, Nicholas WC, Linbeerg SE, Stone CI, Lukaski HC. Rapid smoking: a cautionary note. $\mathcal{F}$ Consult Clin Psychol 1977;45:341-3.

24 Hall RG, Sachs DPR, Hall SM, Benowitz NL. Two-year efficacy and safety of rapid smoking therapy in patients with cardiac and pulmonary disease. $\mathcal{F}$ Consult Clin Psycho 1984;52:574-81

25 Fagerström K-O. Effects of a nicotine titration, daily cigarette consumption, and levels on carbon monoxide, cotinine and nicotine. Psychopharmacology 1982:77:164-7.

26 Mahmarian JJ, Moye LA, Nasser GA, et al A strategy of smoking cessation combined with nicotine patch therapy smoking cessation combined with nicotine patch therapy reduces the extent of exercise-induced
ischemia. $\mathcal{F} \mathrm{Am}$ Coll Cardiol $1997 ; 30: 125-30$.

27 Norregaard J, Tønnesen P, Simonsen K, Petersen L, Säwe U. Smoking habits in relapsed subjects from a smoking cessation trial after one year. $B r \mathcal{F}$ Addict 1992;187:1189 94. 